\title{
Gene therapy for haemophilia: an update on progress in clinical trials
}

Sarah Gilgunn

Clotting factor replacement therapy has proven a highly effective means of treating haemophilia A and B. But treatment involves frequent and lifelong infusion of factor concentrates and is generally prophylactic rather than curative. It is also extremely expensive, associated with inhibitor formation and does not fully abolish the potential for spontaneous bleeding. Gene therapy offers a potential cure for haemophilia, with the possible continuous expression of a clotting factor gene following the administration of a viral vector carrying the appropriate gene. Recent clinical trials of gene therapy for haemophilia have proven positive in selected patients and new studies are underway.

Key words: haemophilia, haemophilia B, Christmas disease, gene therapy

Extensive research into the molecular mechanisms of haemophilia over the past 50 years has greatly advanced our understanding of the disorder, leading to the development of innovative tools for diagnosis alongside pioneering treatments that can minimise the acute and long-term complications associated with the disease [1].

The safety of treatment products is a key concern for the bleeding disorders community. Innovative bioengineered therapies have emerged in the years since many in the community were infected with HIV and hepatitis C (HCV) by clotting factor concentrates in the 1970s and 1980s.

Currently there are many safe and effective products available that have dramatically changed the quality of life (QoL) for people with haemophilia (PWH).

Nevertheless, the 'burden of bleeding' for PWH remains an unresolved concern, one that considerably impact's an individual's QoL. In vitro animal and clinical studies clearly indicate that even only a few haemarthroses into the same joint may cause irreversible structural changes to the joint that impacts both physical function and QoL [2]. Currently, treatment plans aspire to a goal of zero annualised bleeding episodes and through the use of long term individualised prophylactic regimes the development of haemophilic arthropathy can be greatly reduced [2,3].

Although prophylaxis is the recommended standard for treatment, these demanding regimens, often requiring intravenous infusions every other day, are difficult and adherence remains a problem [3]. In addition, convenient access to peripheral veins remains difficult, and many

Sarah Gilgunn, board of directors, Irish Haemophilia Society and molecular biologist, School of Biotechnology, Dublin City University, Glasnevin, Dublin 9, Ireland

Email: sgilgunnamail.dcu.ie

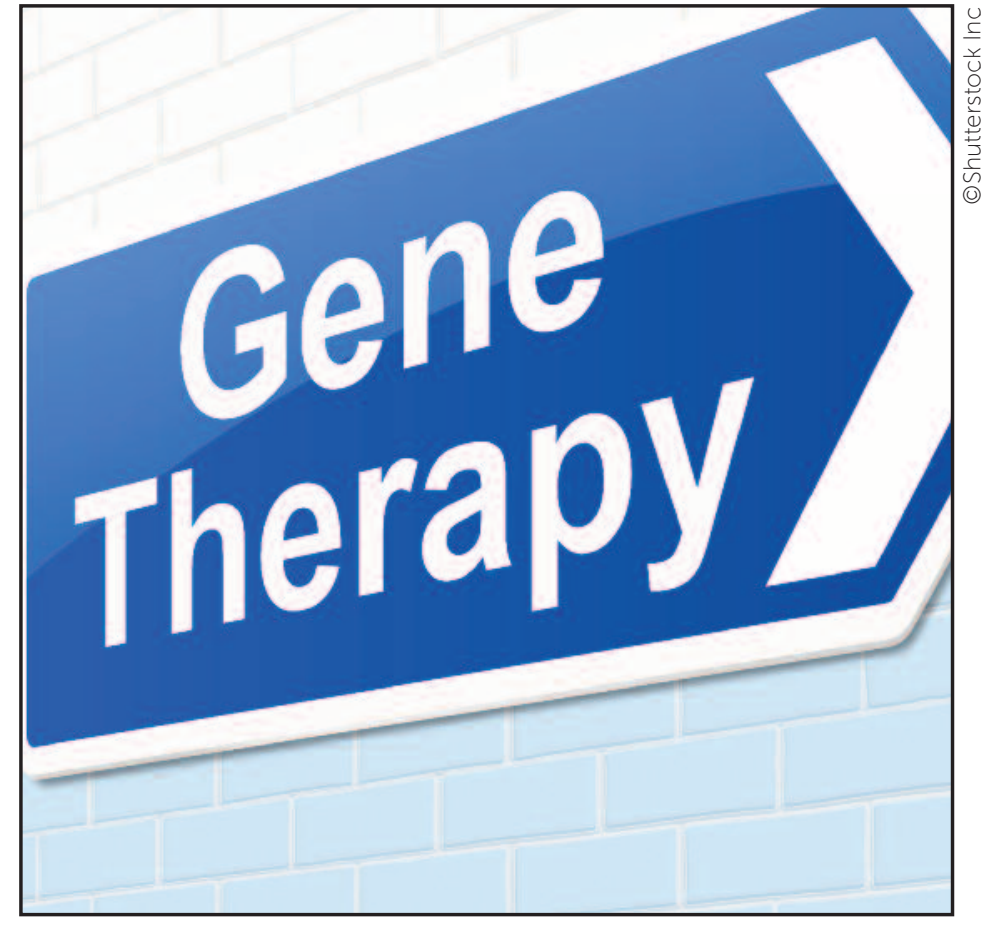

children require use of central venous access devices, which are associated with the medical risks of sepsis and thrombosis [4].

\section{Enter gene therapy}

Haemophilia is a monogenetic disorder, or a single gene disorder, that results from a genetic modification to a single gene occurring in all cells of the body. This makes it a prime candidate for gene therapy [5]. Also, several aspects of the basic biology and pathophysiology of haemophilia make it a paradigmatic genetic disease for correction by gene therapy [6].

The Oxford English Dictionary defines gene therapy as 'the introduction of normal genes into cells in place of missing or defective ones in order to correct genetic disorders'. Gene therapy can theoretically eliminate bleeding episodes by inserting an undamaged gene for factor VIII (FVIII) or factor IX (FIX) into cells of PWH, which can then permanently restore clotting factor production in that patient. Gene therapy therefore offers the potential for permanent cure.

Scientific interest has shifted towards this gene-based approach and there are several, well-validated preclinical studies in animal models demonstrating the therapeutic efficacy of gene therapy for haemophilia. These preclinical studies using haemophilic mouse and dog models 


\section{Table 1: Clinical trials for gene therapy of haemophilia (from Cancio et al, 2013 [1])}

\section{Reference}

- Roth et al, 2001 [11]

\section{Gene and vector}

hFVIII via plasmid DNA

- Powell et al, 2003 [12]

- White et al, 2005 [13]

hFVIII via $\gamma$-retrovirus

hFVIII via HD-Ad

- Manno et al, 2003 [14] hFIX via AAV2

- Manno et al, 2006 [15] hFIX via AAV2

- Nathwani et al, 2011 [16] hFIX via AAV8

\section{Administration and subjects}

Laparoscopic injection of exvivo genetically altered fibrob- efit lasts into the omentum

IV infusion in 13 subjects

IV infusion in 1 subject

IM injection in 8 subjects

Hepatic artery infusion in 7 subjects

IV infusion in 6 subjects

\section{Outcome}

Safe and well tolerated but no long-term ben-

Safe and well tolerated but short-lived circulating FVIII

Severe hepatotoxicity and DIC. No detectable FVIII expression

Safe and well tolerated. Circulating FIX levels $1.5 \%$

\section{Transient transaminitis. FIX expression de- tected but only transiently with loss of expres- sion concurrent with transaminitis. Mild, transient transaminitis in only some high dose patients. Stable FIX expression 1\% for more than 1 year. Effect of transient transaminitis on FIX levels unclear}

achieved sustained elevated clotting factor above the 1-5\% threshold, changing the clinical phenotype from severe to moderate $[7,8]$. Unfortunately, it has proved significantly more problematic than anticipated to translate the early success in animal studies to a clinical setting.

Several early gene therapy trials for haemophilia A and B were initiated using both viral and non-viral vectors, these studies were all first-in-class, that is the first example of the delivery of a specific vector to a particular target tissue. They were generally safe, (no serious adverse events associated with these trials) but none resulted in long-term expression of clotting factor at therapeutic levels [9]. Looking back over two decades of intensive gene therapy research, it can be seen that the early studies have paved the way for the increasingly sophisticated therapies currently under investigation (Table 1).

Many different types of delivery systems (vectors) for transfer of the new gene have been used in the various clinical trials in patients with severe haemophilia A and B; however, four separate trials have used adeno-associated viral (AAV) vectors. These vectors have achieved positive results in a number of clinical and preclinical settings, not just for gene therapy for haemophilia but also disorders such Gaucher's disease, haemochromatosis, and the porphyrias [10]. AAV vectors are a highly attractive vehicle for gene delivery because:

- They lack pathogenicity

- They replicate only in the presence of helper virus

- They are predominantly non-integrating

- They can establish long-term transgene expression following single administration

- Different serotypes are optimal for different tissues $[1,10]$.

In October 2011, Nathwani and colleagues published a seminal paper in which they demonstrated that all participants achieved sustained FIX expression levels above the therapeutic threshold for several months after treatment [16]. This was the first liver-directed AAV trial that resulted in sustained therapeutic FIX levels after gene therapy. The trial, which is taking place at University College London (UCL), has since expanded and 10 patients have enrolled in the study to date, with follow up data for 1-4 years now published [17]. Evidence of sustained FIX expression at $1-6 \%$ in all 10 patients was observed. The majority of these patients have stopped prophylaxis, with significant improvements to QoL: 4 of these patients have reported no spontaneous bleeds since gene therapy treatment. The only adverse reaction reported so far has been an increase in liver enzymes (aspartate aminotransferase and alanine aminotransferase) accompanied by a decrease in FIX. These issues were resolved after a short course of steroids (prednisolone). These preliminary results are extremely encouraging and support further investigational studies, which are ongoing [1, 16-17].

\section{Current trials}

At the Cellular and Molecular Centre for Therapeutics at the Children's Hospital of Philadelphia (CHOP), principle investigator Dr Kathy High and her research team have developed a FIX gene therapy vector similar to the one used in the UCL trial. The vector used is an AAV, serotype 8 (AAV8), which contains a codon-optimised human FIX gene driven by a liver-specific promoter. The group is currently enrolling for a Phase I/II clinical trial to evaluate the safety and tolerability of a single peripheral intravenous administration, in an inter-subject group dose escalation, of AAV8-hFIX19 in adults with severe haemophilia B. Phase I/II clinical trials are often combined to test both efficacy and toxicity (safety, dosage levels, and response to new treatment). The Food and Drug Administration (FDA) definitions of Phase I/II clinical trials are summarised in the panel.

The primary objective of the CHOP trial is to evaluate toxicity related to the administration of AAV8-hFIX19, while 


\section{Panel: Food and Drug Administration (FDA) definitions of Phase I/II clinical trials [18]}

\section{Phase I trials}

Studies that are usually conducted with healthy volunteers and that emphasize safety. The goal is to find out what the drug's most frequent and serious adverse events are and, often, how the drug is metabolized and excreted.

\section{- Phase II trials}

Studies that gather preliminary data on effectiveness (whether the drug works in people who have a certain disease or condition). For example, participants receiving the drug may be compared with similar participants receiving a different treatment, usually an inactive substance (placebo) or a different drug. Safety continues to be evaluated, and short-term adverse events are studied.

secondary objectives are to assess the dose of AAV8$\mathrm{hFIX19}$ required to achieve stable expression of hFIX above $3 \%$ of normal circulating plasma levels, to describe the immune responses to the $\mathrm{hFIX}$ transgene product and AAV capsid proteins following systemic administration of AAV8hFIX19 and to evaluate the safety and toxicity of an immunomodulatory regimen consisting of prednisolone, in those subjects who develop hepatic transaminase elevation after AAV8-hFIX19 administration (K High, personal communication).

\section{Balancing risk and benefit}

It must be clearly noted that the pursuit of gene therapy for haemophilia may be controversial in terms of risk and perceived benefit. The primary objectives of these early phase clinical trials are to evaluate the safety and tolerability of gene therapy as a potential new therapy; therefore it is important to recognise that there is a possibility that long-term factor IX expression will not be achieved in some or even all patients, for reasons that have not yet been uncovered.

Another important topic the haemophilia community needs to consider as these ground-breaking new therapies get underway is the issue of informed consent. Gene therapy is a complex scientific subject and it is important that this material should be presented in a way that is easy for patients considering enrolling in a clinical trial to understand. Informed consent is fundamental to the protection of the rights, safety and wellbeing of patients in clinical research. In order for consent to be valid, patients must be given all the information needed about the proposed research to be able to decide whether they would like to take part [19]. Effective communication between haemophilia treatment centres and the national patient organisations will greatly aid in the informed consent process, assisting patients in making informed decisions. These issues are explored further in the following article by John Morris.

\section{Conclusion}

The current gene therapy studies have illustrated that the dream of a permanent cure is within reach. It has been demonstrated that AAV delivered gene therapy is effective in a relatively restricted subset of the haemophilia patient population (adults with haemophilia B, who are HIVnegative, HCV RNA viral load-negative, and lack neutralizing antibodies to AAV8) [16]. Future clinical studies, such as the CHOP trial, will determine whether this patient group can be safely and effectively expanded and whether the same approach will be effective for haemophilia A.

\section{Disclosures}

The author has advised that she has no interests that might be perceived as posing a conflict or bias.

\section{References}

1. Cancio MI, Reiss UM, Nathwani AC, et al. Developments in the treatment of hemophilia B: focus on emerging gene therapy. Appl Clin Genet 2013; 6: 91-101. doi: 10.2147/TACG.S31928

2. Gringeri A, Ewenstein B, Reininger A. The burden of bleeding in haemophilia: is one bleed too many? Haemophilia 2014; 20(4): 459-63. doi: 10.1111/hae.12375. 3. Schrijvers LH, Uitslager N, Schuurmans MJ, Fischer K.Barriers and motivators of adherence to prophylactic treatment in haemophilia: a systematic review. 355-6. doi: 10.1111/hae.12079.

4. Kaufman RJ, Powell JS. Molecular approaches for improved clotting factors for haemophilia. Hematology Am Soc Hematol Educ Program 2013; 2013: 30-6. doi 10.1182/asheducation-2013.1.30.

5. Chuah MK, Evens H, VandenDriessche T. Gene therapy for hemophilia. J Thromb Haemost 2013; 11 Suppl 1: 99-110. doi: 10.1111/jth.12215.

6. Porada CD, Stem C, Almeida-Porada G. Gene Therapy; The promise of a permanent cure. N C Med J 2013; 74(6): 526-9.

7. Snyder RO, Miao C, Meuse L, et al. Correction of hemophilia B in canine and murine models using recombinant adeno-associated viral vectors. Nat Med 1999 5(1): 64-70.

8. Mount JD, Herzog RW, Tillson DM, et al. Sustained phenotypic correction of hemophilia B dogs with a factor IX null mutation by liver-directed gene therapy. Blood 2002; 99(8): 2670-6.

9. High KA. The gene therapy journey for hemophilia: are we there yet? Blood 2012 9. High KA. The gene therapy journey for hemophilia: are

10. Mingozzi $F$, High KA. Immune responses to AAV vectors: overcoming barriers to successful gene therapy Blood 2013; 122(1): 23-36. doi: 10.1182/blood-2013-01306647.

11. Roth DA, Tawa NE, O'Brien JM, et al. Nonviral transfer of the gene encoding coagulation factor VIII in patients with severe haemophilia A. N Engl J Med 2001 344(23): 1735-1742.

12. Powell JS, Ragni MV, White GC, et al. Phase 1 trial of FVIII gene transfer for severe haemophilia A using a retroviral construct administered by peripheral intravenous infusion. Blood 2003; 102(6): 2038-2045.

13. White GC, Monahan PE. Gene therapy for haemophilia A. In: Lee CA, Berntorp EE, Hoots K, editors. Textbook of Haemophilia. 2nd ed. Hoboken, NJ: WileyBlackwell; 2005

14. Manno CS, Chew AJ, Hutchison S, et al. AAV-mediated factor IX gene transfer to skeletal muscle in patients with severe haemophilia B. Blood 2003; 101(8): 29632972

15. Manno CS, Pierce GF, Arruda VR, et al. Successful transduction of liver in haemophilia by AAV-Factor IX and limitations imposed by the host immune response. Nat Med 2006: 12(3): 342-347.

16. Nathwani AC, Tuddenham EG, Rangarajan S, et al. Adenovirus-associated virus vector-mediated gene transfer in hemophilia B. NEngl JMed2011; 365: 2357-65. doi: 10.1056/NEJMoa1108046.

17. Nathwani AC, Reiss UM, Tuddenham EG, et al. Long-term safety and efficacy of factor IX gene therapy in hemophilia B. NEngl J Med 2014; 371: 1994-2004. doi: 10.1056/NEJMoa1407309.

18. http://www.fda.gov/

19. Pick A, Gilbert K, McCaul J. The role of effective communication in achieving informed consent for clinical trials. Nurs Stand 2014 Nov 5;29(10):45-8. doi: 10.7748/ns.29.10.45.e9443. 